\title{
Hypoxia a consequence of obesity and also a tool to treat excessive weight loss
}

\author{
Pedro González-Muniesa ${ }^{1,2}$, Pablo Quintero ${ }^{3}$, Jacqueline De Andrés ${ }^{4}$, J. Alfredo Martínez ${ }^{1,2 *}$ \\ 1 Department of Nutrition, Food Sciences and Physiology, University of Navarra, Pamplona, Spain \\ 2 CIBERobn Physiopathology of Obesity and Nutrition, Centre of Biomedical Research Network, \\ Madrid, Spain \\ 3 Departamento de Gastroenterología, Pontificia Universidad Católica de Chile, Santiago, Chile \\ 4 Unidad Funcional de Sueño, Hospital Universitario Araba, Vitoria-Gasteiz, Spain
}

* Author to whom correspondence should be addressed: J. Alfredo Martínez

Department of Nutrition, Food Sciences and Physiology, University of Navarra, 31008 Pamplona, Spain

E-Mail: jalfmtz@unav.es; Tel.: +34-948-425-600 (ext. 806424); Fax: +34-948-425-740.

Dear Editor:

We have read with great interest the article by Kong et al [1] about a new strategy to combat obesity and accompanying adverse features, in which normobaric hypoxia training induced more weight loss than normoxia training after 4 weeks on overweight young adults. Such findings complete the view that a modest intermittent hypoxia influence circulating measures of inflammation in healthy humans as reported in a previous issue of Sleep and Breath [2]

Indeed, obesity is a growing public health burden affecting affluent societies as well as transition countries [2] with prevalence rates higher than $30 \%$ in many regions. An energy intake higher than the energy expended for basal metabolic rate, physical activity or thermogenic functions is claimed as the explanation for an excessive fat accumulation, where genetics, obesogenic environmental conditions and other factors such as inflammation and oxidative stress could also be involved [3].

Current therapies for obesity treatment and different approaches for body weight management involve dietary interventions based on energy restriction and/or shifts on macronutrient distribution, physical activity programs, pharmacological prescription or surgical procedures. Unfortunately, none of these therapies, either alone or combined, have been able to be fully satisfactory. Therefore, the search for alternative therapies such as the administration of thermogenic agents, satiating compounds, homeorhetic molecules, etc., are under investigation, including the possibility to use oxygen-based strategies [3].

In this context, abnormal adiposity has been associated not only with self-esteem and image-related psychological problems, but also with insulin resistance, hypertension, osteoarticular problems, cancer, fatty liver disease, etc., where the link between some of these diseases appears to be the mild and chronic inflammation occurring in obesogenic conditions. Thus, one of the mechanisms triggering inflammation has been associated with adipose tissue hypoxia [4] or increased oxygen tension [5], which affect a number of genes such as HIF-1a, MCP-1, IL-6, GLUT-1, ANGPTL-4 and PPAR- $\gamma$ [6] that could be involved as a cause or as a consequence reaction to pathologically low or high oxygen conditions.

Interestingly, as we hypothesized previously [3] and Kong et al have nicely demonstrated in their article [1], hypoxia is not only an ethological factor or a manifestation of disease, but also a therapeutic tool following different patterns (hypoxic exposure, hypoxic training, intermittent hypoxia under hyperbaric and normobaric conditions), which has been used for pre-acclimation in climbers, or to improve athletes performance as well as for heart, respiratory and nervous system diseases or to regulate body weight [7]. Thus, putative compensatory mechanisms and responses to hypoxia has been described for the respiratory 
system (hyperventilation, improvement in respiratory function and increase in lung diffusion capacity), in the cardiovascular system (increase in basal heart rate, peripheral vasodilation, normalization of blood pressure or cardiovascular protection), in cellular and metabolic phenomena (angiogenesis, insulin sensitivity enhancement, enzymatic activation or mitochondrial biogenesis), as well as on body weight management (hypoleptinemia, adrenergic system activation, appetite suppression or serotonin production).

In this sense, in a translational experiment with an animal model we found a reduction in the body weight of rats under hypoxic conditions similar to that reported by Kong et al [1], although a significantly lighter muscle mass in those animals belonging to the hypoxia group was detected (Table 1). Kong et al have been able to avoid this possibly pernicious effect of the hypoxia treatment by implementing a 22 hours of exercise per week program, despite the subjects were on an energy restricted diet [1]. In our experiment the rats did decrease (about 20\%) their muscle mass even though that they were maintained with a high caloric diet mainly due to a macronutrient distribution elevated in lipids. Indeed, it appears important that conjoint exercise and hypoxic conditions are needed to produce a beneficial weight loss without relevant muscle wasting. This view is also in agreement with the experiment carried out by Netzer et al [8], where a low intense physical activity exercise in normobaric hypoxia led to more weight loss in obese people than after normobaric sham hypoxia.

The experiment by Kong et al [1] with their overweight young adults was successful and well-designed, but based on the sedentary lifestyles and deteriorated health status of the Spanish obese adults we have used a different approach (financed by the Spanish MINECO within the EXPLORA subprogram Project SAF2010-11630-E) which is under way. The training protocol consists of two sessions of one hour per week (in normoxia or hypoxia), the diet is only restricted following the Wilkens correction and it is moderately high in protein (30\% of the energy, following previous studies of our group [9]) to preserve lean tissue, and we have also included a third group receiving the same diet counseling as the normoxia and the hypoxia group. This trial is assessing the food intake in hypoxic conditions, since low oxygen supply often impact on appetite as seen in the animal experiment (Table 1). Information from the Kong et al [1] investigation concerning this issue would be of interest to better understand the metabolic outcomes. Interestingly, preliminary data suggest that in addition to changes in body weight, some important benefits on inflammatory and biochemical markers could be achieved by the application of hypoxia within an energy-restricted dietary program based on a moderately high protein intake (about 30 $\%$ of energy).

Summing up, the complex treatment of an excessive adiposity could be improved prescribing exercise under hypoxic conditions to obese subjects to reduce fat accumulation and avoid muscle depletion.

\section{References}

1. Kong Z, Zang Y, Hu Y (2013) Normobaric hypoxia training causes more weight loss than normoxia training after a 4-week residential camp for obese young adults. Sleep Breath. doi 10.1007/s11325-0130922-4

2. Querido JS, Sheel AW, Cheema R, Van Eeden S, Mulgrew AT, Ayas NT (2012) Effects of 10 days of modest intermittent hypoxia on circulating measures of inflammation in healthy humans. Sleep Breath $16: 657-662$

3. Quintero P, Milagro FI, Campion J, Martinez JA (2010) Impact of oxygen availability on body weight management. Med Hypotheses 74:901-907

4. Trayhurn P (2013) Hypoxia and adipose tissue function and dysfunction in obesity. Physiol Rev 93:121

5. Goossens GH, Bizzarri A, Venteclef N, Essers Y, Cleutjens JP, Konings E, Jocken JW, Cajlakovic M, Ribitsch V, Clement K, Blaak EE (2011) Increased adipose tissue oxygen tension in obese compared with lean men is accompanied by insulin resistance, impaired adipose tissue capillarization, and inflammation. Circulation 124:67-76 
6. Quintero P, Gonzalez-Muniesa P, Martinez JA (2012) Influence of different oxygen supply on metabolic markers and gene response in murine adipocytes. J Biol Regul Homeost Agents 26:379-388 7. Urdampilleta A, Gonzalez-Muniesa P, Portillo MP, Martinez JA (2012) Usefulness of combining intermittent hypoxia and physical exercise in the treatment of obesity. J Physiol Biochem 68:289-304 8. Netzer NC, Chytra R, Kupper T (2008) Low intense physical exercise in normobaric hypoxia leads to more weight loss in obese people than low intense physical exercise in normobaric sham hypoxia. Sleep Breath 12:129-134

9. Abete I, Parra D, De Morentin BM, Alfredo Martinez J (2009) Effects of two energy-restricted diets differing in the carbohydrate/protein ratio on weight loss and oxidative changes of obese men. Int J Food Sci Nutr 60 Suppl 3:1-13 